\title{
The EQ-5D - a generic quality of life measure - is a useful instrument to measure quality of life in patients with Parkinson's disease
}

\author{
Anette Schrag, Caroline Selai, Marjan Jahanshahi, Niall P Quinn
}

\begin{abstract}
Objective-To test the feasibility and validity of the EQ-5D (a widely used generic (disease non-specific) quality of life (QoL) instrument which allows comparisons between different patient groups and the general population) to assess QoL in patients with Parkinson's disease.

Methods-All 124 patients with Parkinson's disease seen in a community based study on the prevalence of parkinsonism were asked to complete a QoL battery comprising the EQ-5D, the medical outcome study short form (SF-36), the PDQ39, a disease specific instrument to assess QoL in PD, and the Beck depression inventory. A structured questionnaire interview and a complete neurological examination including the Hoehn and Yahr stage of illness scale, the Schwab and England disability scale, the motor section of the unified Parkinson's disease rating scale (UPDRS), and the mini mental state examination (MMSE) were performed on the same day.
\end{abstract}

Results-The response rate was $78 \%$ and the completion rate of the EQ-5D among responders was $96 \%$. The EQ-5D summary index correlated strongly with the PDQ-39 $(r=-0.75, \mathrm{p}<0.0001)$ as well as the physical score of the SF-36 $(r=0.61$, $\mathbf{p}<0.0001)$. There was a significant correlation of the EQ-5D summary index with disease severity, as measured by the Hoehn and Yahr stage of illness, the Schwab and England disability scale, the motor section of the UPDRS, and the depression score. The EQ-5D summary index also distinguished between patients with and without depression, falls, postural instability, cognitive impairment hallucinations, and those with deterioration of health over the previous year.

Conclusion-The EQ-5D is a feasible and valid instrument to measure QoL in Parkinson's disease and reflects the severity and complications of the disease. (F Neurol Neurosurg Psychiatry 2000;69:67-73)

Keywords: Parkinson's disease; quality of life; EQ-SD; validity

Quality of life is emerging as a critical measure in health care. ${ }^{1}$ Two instruments to measure quality of life in Parkinson's disease are now available. These disease specific instruments are the PDQ-39, a disease specific, well validated, reliable, and sensitive 39 item questionnaire on QoL in Parkinson's disease, ${ }^{2}$ and the PDQL, a validated, reliable, and sensitive measure with 37 items, $^{3}$ which was developed recently and was not available at the beginning of this study. However, with these instruments, a comparison with the general population and other patient groups is not possible due to the disease specificity of their questions ("Due to having Parkinson's disease,. . . have you. . .?"). Generic instruments, which are not disease specific, can therefore be valuable to supplement disease specific QoL instruments to enable comparisons with other patient groups and to assess the impact of the disease on QoL in comparison with the general population. One such generic instrument, the widely used medical outcomes study short form (SF-36) has been used in Parkinson's disease and been shown to have acceptable validity in this patient group. ${ }^{45}$ However, it is relatively long and has lower feasibility in in elderly patient groups with a greater number of missing data. ${ }^{6}$ The EQ $-5 \mathrm{D}^{78}$ is another generic QoL instrument which has been extensively validated and been shown to be sensitive, internally consistent, and reliable in the general population and other patient groups. ${ }^{910}$ Its usefulness in patients with Parkinson's disease has not been examined. It is brief, comprising five questions and a visual analogue scale, which makes it easy to complete and attractive for use in an elderly population. To compare QoL in patients with Parkinson's disease with the general population and other patient groups, we examined the feasibility and validity of the EQ-5D instrument for assessing QoL in Parkinson's disease, in comparison with the disease specific PDQ-39 and the generic SF-36, in a community dwelling population of patients with Parkinson's disease.

Patients and methods

All patients diagnosed with probable Parkinson's disease in a community based study on the prevalence of parkinsonism were included. The methods of patient ascertainment and study design were reported previously. ${ }^{11}$ In brief, the records of 15 general practices in the area of London were screened for patients with a suspicion of Parkinson's disease or parkinsonism, for patients with tremor with onset after the age of 50, and for patients who had ever received antiparkinsonian drugs. Participation rate was high (84\%). Among all 202 patients seen, 124 were diagnosed with probable Parkinson's disease according to the UK Brain Bank criteria. ${ }^{12}$ A structured interview and a complete neurological examination 
including the Hoehn and Yahr scale (range $0--5$; the highest disease severity indicated by $5),{ }^{13}$ the Schwab and England disability scale (range 0 to $100 \%$; the greatest independence indicated by $100 \%),{ }^{14}$ the motor section of the unified Parkinson's disease rating scale (UPDRS; range 0-56; the highest disease severity indicated by 56$)^{15}$ and a mini mental state examination (MMSE; range 0-30; the best cognitive performance indicated by 30$)^{16}$ were performed by a neurologist on the same day. The structured interview included questions about clinical features such as presence/ absence of falls, forgetfulness, on-off fluctuations, dyskinesias, and hallucinations. All eligible patients were asked to complete the EQ-5D, the PDQ-39, the SF-36, and the Beck depression inventory, and a question on whether their general health in comparison with 1 year ago was improved, much the same, or worse, and to return them in a stamped, addressed envelope.

\section{QOL INSTRUMENTS}

The EQ-5D

The EQ-5D comprises five questions on mobility, self care, pain, usual activities, and psychological status with three possible answers for each item $(1=$ no problem, $2=$ moderate problem, 3=severe problem; see appendix). ${ }^{78} \mathrm{~A}$ summary index with a maximum score of 1 can be derived from these five dimensions by conversion with a table of scores. The maximum score of 1 indicates the best health state, by contrast with the scores of individual questions, where higher scores indicate more severe or frequent problems. In addition, there is a visual analogue scale (VAS) to indicate the general health status with 100 indicating the best health status.

\section{The PDQ-39}

The PDQ- $39^{2}$ comprises 39 questions each with five different answer options, from which eight subscores (mobility, activities of daily living, emotional wellbeing, stigma, social support, cognition, communication, and bodily discomfort) and one summary index can be calculated. The maximum score of 100 indicates the worst level of problem.

\section{The SF-36}

The SF- $36^{17}$ comprises 36 questions. Eight different subscores (physical and social functioning, physical and emotional role limitations, mental health, energy, pain, and general health perceptions), and a physical and mental summary score can be derived from these. The maximum score of 100 indicates the best possible health state.

Feasibility-Feasibility was determined by the number of missing responses in each instrument.

Validity - The validity of an instrument is the extent to which it measures what it claims to measure. Different types of validity can be assessed. Criterion validity is the correlation of a scale with some other measure of the disorder under study, ideally a "gold standard" which has been used and accepted in the field. In this study, we examined the correlations between the EQ-5D and other measures assessing similar aspects of wellbeing. Construct validation is an ongoing process whereby hypothetical "constructs" are tested. One such hypothesis might be that higher disease severity would correlate with lower scores indicating worse QOL. ${ }^{18}$ We tested the a priori hypothesis that patients with more severe Parkinson's disease, those with longer duration of disease and those with complications of disease and treatment would have worse QoL than those with early disease without these complications.

\section{STATISTICAL ANALYSIS}

The subscores and summary scores were calculated according to the respective scoring algorithms. We selected non-parametric tests for comparisons because of the ordinal nature of the scales. Group differences were analysed by the Mann-Whitney and Kruskal-Wallis tests and associations between scales with Spearman rank correlations. Statistical comparisons were performed between all variables that may hypothetically influence QoL scores, such as the duration and severity of disease, the presence of complications of advancing disease or treatment, or those that have been reported to be associated with reduced QoL, such as age or socioeconomic group. ${ }^{19}$ The validity of the EQ-5D was examined in several ways: firstly, by measuring the association between the EQ-5D summary index, the subscores, and the visual analogue score with the clinical ratings of disease severity in Parkinson's disease; secondly, by examining its correlation with the generic (SF-36) and the disease-specific (PDQ-39) measures of QoL; thirdly, by determining whether the EQ-5D successfully differentiated between patients with and without features of advanced disease and complications of its treatment, such as those with or without falls, dyskinesias, and dementia. Because we performed many comparisons we applied the Bonferroni correction. Given that we had 108 comparisons in the first part of the analysis, 133 in the second part of the analysis, and 30 in the third part, for an overall level of significance of $\alpha=0.05$, the corrected $p$ values for consideration in individual comparisons become $\mathrm{p}=0.0005, \mathrm{p}=0.00038$, and $\mathrm{p}=0.0017$ respectively. All statistical analyses were performed with SPSS for Windows. ${ }^{20}$

\section{Results}

PROPERTIES OF THE EQ-5D

The median EQ-5D score was 0.62 (minimum -0.59 and maximum 1.0) with an interquartile range of 0.29 . To examine whether the EQ-5D adequately reflects patient diversity, we inspected the distribution of the EQ-5D scores using histogram plots, which disclosed a similar distribution for the EQ-5D, SF-36 physical summary, Schwab and England and Hoehn and Yahr scores, with most patients in the low to moderate ranges of disease severity, disability, and impairment of QoL score. 
Table 1 Spearman correlation coefficients of different quality of life measures with clinical scales and disease duration

\begin{tabular}{|c|c|c|c|c|c|c|}
\hline & $\begin{array}{l}\text { Hoehn and } \\
\text { Yahr stage }\end{array}$ & $\begin{array}{l}\text { Schwab and } \\
\text { England } \\
\text { disability score }\end{array}$ & $\begin{array}{l}\text { UPDRS } \\
\text { motor score }\end{array}$ & $\begin{array}{l}\text { Disease } \\
\text { duration }\end{array}$ & $\begin{array}{l}\text { Beck } \\
\text { depression } \\
\text { inventory }\end{array}$ & $\begin{array}{l}\text { Mini mental } \\
\text { state score }\end{array}$ \\
\hline \multicolumn{7}{|l|}{ EQ-5D: } \\
\hline Mobility & $0.70^{\star}$ & $-0.65^{\star}$ & $0.40^{\star}$ & 0.35 & $0.45^{\star}$ & -0.37 \\
\hline Self care & $0.51^{\star}$ & $-0.50^{\star}$ & 0.36 & 0.25 & $0.54^{\star}$ & -0.17 \\
\hline Ususal activities & $0.65^{\star}$ & $-0.65^{\star}$ & $0.40^{\star}$ & 0.29 & $0.59^{\star}$ & -0.37 \\
\hline Pain & $0.49^{\star}$ & $-0.47^{\star}$ & 0.31 & 0.12 & $0.45^{\star}$ & -0.24 \\
\hline Psychological functioning & $0.41^{\star}$ & -0.37 & 0.26 & 0.04 & $0.55^{\star}$ & -0.26 \\
\hline Summary index & $-0.68^{\star}$ & $0.66^{\star}$ & $-0.40^{\star}$ & -0.21 & $-0.63^{\star}$ & 0.35 \\
\hline Visual analogue scale & -0.32 & $0.42^{\star}$ & -0.20 & -0.03 & $-0.63^{\star}$ & 0.23 \\
\hline \multicolumn{7}{|l|}{ PDQ-39: } \\
\hline Activities of daily living & $0.63^{\star}$ & $-0.55^{\star}$ & $0.52^{\star}$ & 0.30 & $0.53^{\star}$ & -0.23 \\
\hline Bodily discomfort & 0.36 & $-0.49^{\star}$ & 0.28 & 0.16 & $0.53^{\star}$ & -0.31 \\
\hline Cognition & $0.43^{\star}$ & $-0.44^{\star}$ & 0.26 & 0.15 & $0.67^{\star}$ & -0.15 \\
\hline Communication & $0.49^{\star}$ & $-0.50^{\star}$ & 0.31 & 0.19 & $0.47^{\star}$ & -0.23 \\
\hline Emotional wellbeing & $0.40^{\star}$ & $-0.51^{\star}$ & 0.20 & 0.04 & $0.57^{\star}$ & -0.36 \\
\hline Mobility & $0.69^{\star}$ & $-0.68^{\star}$ & $0.45^{\star}$ & 0.18 & $0.54^{\star}$ & $-0.39^{\star}$ \\
\hline Social support & 0.22 & -0.36 & 0.10 & -0.01 & 0.32 & -0.09 \\
\hline Stigma & 0.26 & -0.36 & $0.39^{\star}$ & 0.10 & $0.44^{\star}$ & -0.10 \\
\hline Summary index & $0.60^{\star}$ & $-0.65^{\star}$ & $0.41^{\star}$ & 0.18 & $0.68^{\star}$ & -0.32 \\
\hline \multicolumn{7}{|l|}{ SF-36: } \\
\hline Physical summary score & $-0.50^{\star}$ & $0.49^{\star}$ & $-0.41^{\star}$ & -0.21 & $-0.42^{\star}$ & 0.08 \\
\hline Mental summary score & -0.25 & 0.27 & -0.16 & 0.06 & $-0.49^{\star}$ & 0.35 \\
\hline
\end{tabular}

${ }^{\star} \mathrm{p}<0.0005$

UPDRS=Unified Parkinson's disease rating scale.

FEASIBILITY

Ninety seven of the 124 patients returned the questionnaires, which constitutes a response rate of $78 \%$. Their mean age was 73 (SD 11.3), ranging from 36 to 94 years with a mean disease duration of 5.8 (SD 4.9) ranging from 0.2 to 25 years, and a mean onset age of 67.6 (SD 12.5) ranging from 23.7 to 93.5 years. There were 50 men and 47 women who responded, mean Hoehn and Yahr stage was 2.4 (SD 0.9) ranging from 1 to 5), and mean MMSE was 27.5 (out of 30; SD 3.6). There were $20(4.1 \%)$ missing responses on the EQ-5D and six (6.2\%) patients had not completed the VAS. On the PDQ-3 9, 180 answers were missing $(4.8 \%)$, and on the SF-36 286 answers (8.2\%) were not completed. There was no statistical difference between responders and non-responders for age, disease duration, sex, Hoehn and Yahr stage, Schwab and England score, or MMSE score.

\section{VALIDITY}

Correlation with ratings of disease severity (table 1) Given the large number of comparisons, we decided to apply the Bonferroni correction. For this part of the analysis, correlations were considered significant when $\mathrm{p}<0.0005$.

$E Q-5 D$ summary index-As expected, lower EQ-5D summary index scores correlated significantly with greater disease severity as measured by Hoehn and Yahr stages $(n=77$, $r=-0.68, \mathrm{p}<0.0001$; table 1 and 2), with lower independence scores, as measured with the Schwab and England disability scale $(n=77$, $r=0.66, \mathrm{p}<0.0001)$ and with higher BDI scores $(\mathrm{n}=77, r=-0.63, \mathrm{p}<0.0001$; table 1$)$. Most dimensions of QoL, as measured in the subscores, also correlated significantly with both disease scales but, as expected, in the opposite direction. Higher scores on the motor section of the UPDRS correlated with lower EQ-5D summary scores, but lower MMSE scores or longer disease duration did not correlate significantly with lower EQ-5D summary or sub-scores.
Visual analogue scale-The correlation of the VAS with measures of disease severity were in the same direction but less strong than those obtained for the EQ-5D summary index. The VAS correlated significantly with the degree of independence quantified with the Schwab and England scale $(n=91, r=0.42, p<0.0001)$ and negatively with the depression score as measured by the BDI ( $n=9 \quad 1, r=-0.63$, $\mathrm{p}<0.0001$ ), but not with the motor section of the UPDRS and the Hoehn and Yahr scale.

Correlation with other QoL scales (table 3)

According to the Bonferroni method, for this part of the analysis correlations were considered significant when $\mathrm{p}<0.00038$. The EQ-5D summary index correlated strongly and negatively with the PDQ-39 summary index ( $n=73$, $r=-0.75, \mathrm{p}<0.0001$ ) and most PDQ-39 subscores, and positively with the SF-36 physical summary score and most subscores (table 3). As expected, the strongest associations were between the EQ-5D and PDQ-39 or SF-36 subscores of both instruments that measure similar aspects of QoL, indicating good convergent validity. The VAS of the EQ-5D correlated significantly with all the SF-36 (positively) and PDQ-39 scores (negatively) with correlations ranging from 0.40 to 0.62 , except the "social functioning" subscore of the PDQ-39, and the "emotional role" subscore of the SF-36, which did not correlate significantly with any subscale of the EQ-5D.

\section{Sensitivity to clinical features of Parkinson's} disease

According to the Bonferroni method, in this part of the analysis correlations were considered significant when $\mathrm{p}<0.0011$.

$E Q-5 D$ summary index and visual analogue scale-Using a cut off score of 9 to distinguish mild from moderate/severe self reported depression as measured on the BDI, the EQ-5D summary index was significantly different for patients with and without depression $(\mathrm{p}<0.0001) .{ }^{21}$ Patients with cognitive deficits as indicated by an MMSE less than 25 had 
Table 2 QoL scores compared between groups of patients identified by the presence or absence of clinical features or complications that significantly impair quality of life

\begin{tabular}{|c|c|c|c|c|c|c|c|c|c|c|}
\hline & \multicolumn{2}{|c|}{$E Q-5 D S I$} & \multicolumn{2}{|c|}{$E Q-5 D$ VAS } & \multicolumn{2}{|c|}{ PD39 SI } & \multicolumn{2}{|c|}{$\begin{array}{l}\text { SF36 Physical } \\
\text { summary score }\end{array}$} & \multicolumn{2}{|c|}{$\begin{array}{l}\text { SF36 Mental } \\
\text { summary score }\end{array}$} \\
\hline & (mean) & p Value & (mean) & p Value & (mean) & $p$ Value & (mean) & $p$ Value & (mean) & p Value \\
\hline \multicolumn{11}{|c|}{ Depression (BDI \pm 9$)$ : } \\
\hline Present & 0.4 & $<0.0001$ & 54.9 & $<0.0001$ & 39.0 & $<0.0001$ & 31.3 & 0.0001 & 43.6 & $<0.0001$ \\
\hline Absent & 0.7 & & 76.7 & & 16.7 & & 40.3 & & 52.0 & \\
\hline \multicolumn{11}{|c|}{ Mini mental score <25: } \\
\hline Present & 0.2 & $<0.0001$ & 51.1 & 0.007 & 49.4 & $<0.0001$ & 29.7 & 0.05 & 40.9 & 0.006 \\
\hline Absent & 0.7 & & 69.0 & & 24.4 & & 37.2 & & 49.5 & \\
\hline \multicolumn{11}{|l|}{ Falls:* } \\
\hline Present & 0.4 & $<0.0001$ & 58.6 & 0.006 & 36.2 & $<0.0001$ & 31.4 & 0.006 & 46.2 & 0.3 \\
\hline Absent & 0.8 & & 71.7 & & 21.4 & & 39.8 & & 48.5 & \\
\hline \multicolumn{11}{|l|}{ Postural instability: } \\
\hline Present & 0.4 & $<0.0001$ & 57.2 & $<0.0001$ & 33.7 & 0.001 & 32.2 & 0.0007 & 47.8 & 0.7 \\
\hline Absent & 0.8 & & 77.6 & & 18.7 & & 42.3 & & 46.3 & \\
\hline \multicolumn{11}{|c|}{ Change in health over the previous year: ${ }^{\star}$} \\
\hline Improvement $\dagger$ & 0.6 & 0.7 & 70.5 & 0.9 & 16.8 & 0.2 & 36.2 & 0.8 & 53.4 & 0.2 \\
\hline No changeł & 0.6 & $<0.0001$ & 70.9 & $<0.0001$ & 25.1 & 0.0001 & 37.0 & 0.003 & 48.0 & 0.1 \\
\hline Worsening $\llbracket$ & 0.3 & 0.0002 & 49.1 & 0.0001 & 43.4 & 0.0002 & 30.0 & 0.01 & 43.3 & 0.1 \\
\hline \multicolumn{11}{|c|}{ Hoehn and Yahr stage: $§$} \\
\hline I & 0.9 & & 78.5 & & 16.6 & & 46.2 & & 49.5 & \\
\hline II & 0.6 & & 66.8 & & 28.1 & & 35.5 & & 48.5 & \\
\hline III & 0.3 & & 53.8 & & 40.8 & & 28.2 & & 43.9 & \\
\hline $\mathrm{IV} / \mathrm{V}$ & 0.2 & 0.0001 & 52.0 & 0.02 & 38.6 & 0.0001 & 22.0 & 0.005 & 35.3 & 0.05 \\
\hline
\end{tabular}

VAS=Visual analogue scale; SI=summary index; *self reported; timprovement compared with no change, łno change compared with worsening, \Kruskall -Wallis test for all three/four levels; all other comparisons were Mann-Whitney tests

significantly lower EQ-5D scores than the group without cognitive deficits $(p<0.0001)$. The features of the illness which had a significant impact on EQ-5D scores were presence or absence of falls $(p<0.0001)$ and postural instability $(\mathrm{p}<0.0001)$, and on-off fluctuations and experience of hallucinations tended to influence EQ-5D scores $(p=0.046$ and $p=0.007$, respectively). In addition, patients who thought that their general health had deteriorated over the past year had significantly worse EQ-5D scores than those whose health had subjectively remained much the same $(\mathrm{p}<0.0001)$. By contrast, age (being older or younger than 70 years) had no significant impact on EQ-5D scores. There was no significant difference in EQ-5D scores between those patients who considered themselves improved compared with 1 year before and those who had not, but this may have been due to the few patients $(n=6)$ who had noticed an improvement. Presence or absence of dyskinesias, gait impairment, incontinence, orthostatic symptoms, insomnia, visual disturbances, pain, speech, or swallowing impairment or a family history of Parkinson's disease did not differentiate significantly between patients with higher or lower QoL scores, as measured on any of the QoL instruments.

The visual analogue scale was less sensitive to these disease related features and only significantly differentiated groups with or without depression $(\mathrm{p}<0.0001)$, postural instability $(\mathrm{p}<0.0001)$, and deterioration of general health over the past year $(\mathrm{p}<0.0001)$, and tended to differentiate patients with falls and those with cognitive deficits (table 2).

Comparisons with other QoL measures-The correlation of the EQ-5D summary index and its subscores with the clinical scales was very

Table 3 Spearman correlation coefficients of the EQ-5D summary score, sub-scores and visual analogue scale with the generic (SF 36) and the disease specific (PDQ-39) quality of life measures

\begin{tabular}{|c|c|c|c|c|c|c|c|}
\hline & \multicolumn{7}{|l|}{$E Q-5 D$} \\
\hline & Mobility & Self care & $\begin{array}{l}\text { Usual } \\
\text { activities }\end{array}$ & Pain & Anxiety & $\begin{array}{l}\text { Summary } \\
\text { index }\end{array}$ & $\begin{array}{l}\text { Visual } \\
\text { analogue } \\
\text { score }\end{array}$ \\
\hline \multicolumn{8}{|l|}{ PDQ 39 SI: } \\
\hline Mobility & $0.73^{\star}$ & $0.59^{\star}$ & $0.67^{\star}$ & $0.54^{\star}$ & $0.47^{\star}$ & $-0.74^{\star}$ & $-0.56^{\star}$ \\
\hline ADL & $0.65^{\star}$ & $0.72^{\star}$ & $0.65^{\star}$ & 0.37 & 0.38 & $-0.63^{\star}$ & $-0.41^{\star}$ \\
\hline Emotional & $0.54^{\star}$ & $0.42^{\star}$ & $0.46^{\star}$ & 0.37 & $0.50^{\star}$ & $-0.54^{\star}$ & $-0.43^{\star}$ \\
\hline Stigma & 0.34 & 0.30 & 0.33 & 0.35 & $0.48^{\star}$ & $-0.41^{\star}$ & $-0.47^{\star}$ \\
\hline Social functioning & 0.26 & 0.28 & 0.28 & 0.31 & 0.26 & -0.35 & -0.30 \\
\hline Cognitions & $0.49^{\star}$ & $0.51^{\star}$ & $0.58^{\star}$ & 0.35 & $0.45^{\star}$ & $-0.55^{\star}$ & $-0.47^{\star}$ \\
\hline Communication & $0.53^{\star}$ & $0.58^{\star}$ & $0.57^{\star}$ & 0.34 & 0.28 & $-0.56^{\star}$ & $-0.42^{\star}$ \\
\hline Bodily & 0.40 & 0.35 & $0.45^{\star}$ & $0.47^{\star}$ & 0.40 & $-0.55^{\star}$ & $-0.51^{\star}$ \\
\hline Summary score & $0.69^{\star}$ & $0.63^{\star}$ & $0.68^{\star}$ & $0.54^{\star}$ & $0.55^{\star}$ & $-0.75^{\star}$ & $-0.60^{\star}$ \\
\hline \multicolumn{8}{|l|}{ SF-36: } \\
\hline Physical functioning & $-0.58^{\star}$ & $-0.44^{\star}$ & $-0.52^{\star}$ & $-0.42^{\star}$ & -0.36 & $0.57^{\star}$ & $0.47^{\star}$ \\
\hline Physical role & $-0.42^{\star}$ & -0.18 & -0.40 & -0.40 & -0.27 & $0.43^{\star}$ & $0.43^{\star}$ \\
\hline Bodily pain & -0.35 & -0.33 & -0.36 & $-0.59^{\star}$ & -0.32 & $0.56^{\star}$ & $0.54^{\star}$ \\
\hline General health & -0.37 & -0.40 & $-0.48^{\star}$ & -0.36 & -0.35 & $0.47^{\star}$ & $0.62^{\star}$ \\
\hline Vitality & -0.39 & $-0.46^{\star}$ & $-0.48^{\star}$ & -0.34 & $-0.46^{\star}$ & $0.52^{\star}$ & $0.49^{\star}$ \\
\hline Social functioning & $-0.56^{\star}$ & $-0.52^{\star}$ & $-0.52^{\star}$ & $-0.46^{\star}$ & $-0.46^{\star}$ & $0.59^{\star}$ & $0.53^{\star}$ \\
\hline Emotional role & -0.19 & -0.13 & -0.19 & -0.11 & -0.21 & 0.20 & 0.25 \\
\hline Mental health & -0.32 & $-0.43^{\star}$ & $-0.45^{\star}$ & -0.36 & $-0.53^{\star}$ & $0.50^{\star}$ & $0.49^{\star}$ \\
\hline Physical score & $-0.52^{\star}$ & $-0.41^{\star}$ & $-0.51^{\star}$ & $-0.53^{\star}$ & -0.31 & $0.61^{\star}$ & $0.55^{\star}$ \\
\hline Mental score & -0.25 & -0.33 & -0.34 & -0.20 & $-0.41^{\star}$ & 0.35 & 0.40 \\
\hline
\end{tabular}

$\mathrm{ADL}=$ Activities of daily living

${ }^{\star} \mathrm{p}<0.00038$. 
similar to that of the PDQ-39 and the SF-36 summary scores (table 1 ). The correlation of the VAS with these scales, on the other hand, was weaker than that of the PDQ-39 summary index and SF-36 physical summary, except its correlation with the BDI. However, the association of the VAS of the EQ-5D with the clinical measures was better than the correlation of the mental summary of the SF-36 with these scales. Furthermore, the EQ-5D significantly differentiated between patients with and without particular clinical features such as postural instability, worsening of health over the past year, or depression as well as the PDQ-39 and better than the SF-36 physical score (table 2).

\section{Discussion}

We found that the EQ-5D summary index had good feasibility and validity in this community dwelling population of patients with Parkinson's disease. It correlated moderately with the measures of disease severity and disability as well as with depression, reflecting its sensitivity to disease severity coupled with sufficient distinctiveness. It also correlated strongly with the disease specific PDQ-39 summary index and its subscores and, to a lesser degree, with the physical score of the generic health status instrument SF-36. Its sensitivity to the clinical features of Parkinson's disease was similar to that of the disease specific instrument, whereas the SF-36 was less sensitive. These results are similar to those reported for the disease specific QoL instrument, the PDQ-39. ${ }^{2}$ The validity of the EQ-5D summary index in this sample of patients with Parkinson's disease was high and better than reported for other disorders such as epilepsy, ${ }^{22}$ or lower limb ischaemia, ${ }^{23}$ and comparable to that of the disease specific PDQ-39. A possible explanation for this finding could be that the correlation of the PDQ-39 with clinical scales was poorer than in previous studies, which were either clinic based or used a selected sample (members of the Parkinson's disease society). ${ }^{2}$ However, the correlation of the PDQ-39 and the SF-36 with the Hoehn and Yahr and other clinical scales found in this study was very similar to previously reported results, ${ }^{44}$ confirming their validity in an unselected sample of patients with Parkinson's disease. Therefore, at first sight it may seem surprising that a non-specific generic measure which only comprises five questions with three levels of response option correlated well with the clinical scales. However, it should be noted that these five questions cover five core areas which are particularly important in Parkinson's disease: mobility, self care, usual activities, pain, and anxiety/depression. They represent the aspects of life most affected by Parkinson's disease and its typical features. Other features such as stigma, social support, communication, and cognition are not covered, but the areas most relevant for QoL in Parkinson's disease, as found by Fitzpatrick et $a l,{ }^{24}$ are also those covered in the EQ-5D: difficulty with leisure activities, difficulty looking after the home, problems writing clearly (covered in "usual activities"), difficulties getting around in public places, problems walking half a mile, difficulty carrying shopping bags (covered in "mobility"), problems in doing up buttons or shoelaces (covered in "self care"), worrying about the future (covered in anxiety/ depression) and bodily aches and pains (covered in "pain"). The EQ-5D may be a particularly suitable generic instrument for patients with Parkinson's disease as it is a very mobility based questionnaire. It may also be more valid in this patient group than, for example, in patients with epilepsy or headache as there are no disease free intervals which could distort the true extent of the problem when asked for the "health state today". It may also be more suitable for patients with Parkinson's disease than for patients with congenital or early onset disorders as Parkinson's disease usually starts late in life so that patients can easily compare their current to their previous health state. The VAS part of the EQ-5D, although easy to complete, was not as strongly correlated with clinical measures or sensitive to the presence or absence of clinical features. On the other hand, it correlated highly with depression. The SF-36 was a generally less sensitive measure (lower correlation with clinical scales, and poorer differentiation between patients with and without features of advanced disease) than the EQ-5D, summary index and the PDQ-39, and additionally had a higher percentage of missing answers. Moreover, patients often added comments to its questions as they did not think that these were appropriately reflecting their problems, such as

"I have arthritis and can therefore not walk long distances".

A shortcoming of this study is that we did not formally assess the EQ-5D's sensitivity to change, which is important if a QoL instrument is to be used in clinical trials to test effectiveness of treatment. This was a cross sectional study with only one evaluation of QoL. However, patients who reported deterioration of their general health over the past year had significantly worse QoL scores than those whose health had not deteriorated or improved, indicating the instrument's ability to reflect change in disease severity. Furthermore, it may be argued that a selection bias towards patients with less severe disease or better cognitive scores in those who completed the questionnaire cannot be excluded. However, disease severity and cognitive scores did not differ between responders and non-responders (all of whom were examined), and the participation rate in the overall community based prevalence study was high $(84 \%)$. Nevertheless, those who declined participation were significantly older than those who were seen $(\mathrm{p}<0.001)$, and such a bias can therefore not be entirely excluded. Finally, the multitude of comparisons makes the study, despite application of the Bonferroni method, an exploratory one, and the results will later have to be confirmed in a subsequent set of data.

We have shown the EQ-5D to be feasible and valid in a community dwelling population of patients with Parkinson's disease. The findings of this study suggest that the EQ-5D summary score differs between patients grouped by cer- 
tain clinical features, and correlates well with clinical scales, similar to the disease specific PDQ-39 summary score. However, the significance of individual comparisons will have to be confirmed on new sets of patients, and its sen- sitivity to change needs to be assessed in further studies.

This study was made possible by a grant from SmithKline Beecham Pharmaceuticals.

\section{Appendix: The EQ-5D instrument}

By placing a tick in one box in each group below, please indicate which statements best describe your own health state today.

Mobility

I have no problems in walking about

I have some problems in walking about

I am confined to bed

Best imaginable

Self-Care

I have no problems with self-care

I have some problems washing or dressing myself

I am unable to wash or dress myself

Usual Activities (eg work, study, housework, family or leisure activities)

I have no problems with performing my usual activities I have some problems with performing my usual activities

I am unable to perform my usual activities

\section{Pain/Discomfort}

I have no pain or discomfort

I have moderate pain or discomfort

I have extreme pain or discomfort

\section{Anxiety/Depression}

I am not anxious or depressed

I am moderately anxious or depressed

I am extremely anxious or depressed

To help people say how good or bad a health state is, we have drawn a scale (rather like a thermometer) on which the best state you can imagine is marked 100 and the worst state you can imagine is marked 0 .

We would like you to indicate on this scale how good or bad your own health is today, in your opinion. Please do this by drawing a line from the box below to whichever point on the scale indicates how good or bad your health state is today.

Note: The actual EQ-5D instrument spreads over two pages with the"thermometer" and explanation on page 2. To save journal space in this paper it has been compressed into one page.

\section{Your own \\ health state \\ today}

1 Devinsky O. Outcome research in neurology: incorporating health-related quality of life. Ann Neurol 1995;37: 141-2.

2 Peto V, Jenkinson C, Fitzpatrick R, et al. The development and validation of a short measure of functioning and well being for individuals with Parkinson's disease. Qual Life Res 1995;4:241-8.

3 de Boer AG, Wijker W, Speelman JD, et al. Quality of life in patients with Parkinson's disease: development of a questionnaire. I Neurol Neurosurg Psychiatry 1996;61: $70-4$.

4 Rubenstein LM, Voelker MD, Chrischilles EA, et al. The usefulness of the functional status questionnaire and medi- cal outcomes study short form in Parkinson's disease research. Qual Life Res 1998;7:279-90.

5 Jenkinson C, Peto V, Fitzpatrick R, et al. Self-reported functioning and well-being in patients with Parkinson's disease: comparison of the short-form health survey (SF-36) and the Parkinson's disease questionnaire (PDQ-39). Age Ageing 1995;24:505-9.

6 Brazier JE, Waiters SJ, Nicholl JP, et al. Using the SF-36 and Euroqol on an elderly population. Oual Life Res 1 996;5:195-204.

7 EuroQoL Group. EuroQoL: a new facility for the measurement of heath-related quality of life. Health Policy 1990;16: 199-208. 
8 Brooks R, with the EuroQol Group. EuroQol: the current state of play. Health Policy 1996;37:53-72

Dorman PJ, Waddell F, Slattery J, et al. Is the EuroOol a valid measure of health-related quality of life after stroke? Stroke 1997;28:1876-82

10 Hurst NP, Jobanputra P, Hunter M, et al. Validity of Euroqol-a generic health status instrument in patients with rheumatoid arthritis. Economic and health outcomes research group. Br f Rheumatol 1994;33:655-62.

11 Schrag A, Ben-Shlomo Y, Quinn NP. Prevalence of progressive supranuclear palsy and multiple system atrophy: a cross-sectional study. Lancet 1999;354:1771-75.

12 Gibb WR, Lees AJ. The relevance of the Lewy body to the pathogenesis of idiopathic Parkinson's disease. F Neurol Neurosurg Psychiatry 1988;51:745-52.

13 Hoehn MM, Yahr MD. Parkinsonism: onset, progression and mortality. Neurology 1967;17:427-42.

14 Schwab RS, England AC. Projection technique for evaluating surgery in Parkinson's disease. In: Gillingham FJ, Doning surgery in Parkinson's disease. In: Gillingham FJ, DonEdinburgh: Livingstone, 1969:152-7.

15 Fahn S, Elton RL, members of the UPDRS Development Committee. Unified Parkinson's disease rating scale. In Fahn S, Marsden CD, Calne DB, et al, eds. Recent developments in Parkinson's disease. Vol 2. Florham Park, NJ: MacMillan Healthcare Information, 1987:153-63.
16 Folstein MF, Folstein SE, McHugh PR. Mini-mental state. A practical method for grading the cognitive state of patients for the clinician. F Psychiatr Res 1975;12:189-98.

17 McHorney CA, Ware JE Jr, Raczek AE. The MOS 36-item short-form health survey (SF-36): II. Psychometric and clinical tests of validity in measuring physical and mental health constructs. Med Care 1993;31:247-63.

18 Streiner DL, Norman GR. Health status measurement. 2nd ed. Oxford: Oxford University Press, 1995.

19 Kind P, Dolan P, Gudex C, et al. Variations in population health status: results from a United Kingdom national questionnaire survey. BMF 1998;316:736-41.

20 SPSS. SPSS for Windows, Rel 8.0.0. Chicago: SPSS, 1997.

21 Beck AT, Ward C, Mendelson M, et al. An inventory for measuring depression. Arch Gen Psychiatry 1961;4:561-7

22 Selai CE, Elstner K, Trimble MR. Quality of life pre-and post epilepsy surgery. Epilepsy Res 2000;38:67-74.

23 Chetter IC, Spark JI, Dolan P, et al. Quality of life analysis in patients with lower limb ischaemia: suggestions for European standardisation. Eur f Vasc Endovasc Surg 1997; 13:597-604.

24 Fitzpatrick R, Peto V, Jenkinson C, et al. Health-related quality of life in Parkinson's disease: a study of outpatient clinic attendees. Mov Disord 1997;12:916-22. 